\title{
Idiopathic pediatric chronic kidney disease: can genomic technology crack the case?
}

\author{
Martin R. Pollak \\ Beth Israel Deaconess Medical Center, Boston, Massachusetts, USA.
}

\begin{abstract}
In children, chronic kidney disease (CKD) that results from structural abnormalities and glomerular injury is readily diagnosed; however, most cases of pediatric CKD are of unknown etiology. In this issue of the $J C I$, Verbitsky and colleagues used chromosomal microarrays to evaluate genomic variation in children with CKD. Compared with control individuals, a substantial proportion of children with idiopathic CKD had clearly identifiable genomic imbalances. Moreover, in some cases, detailed analysis of these imbalances identified pathogenic alterations that were unsuspected based on clinical presentation. The results of this study support genomewide evaluation for pediatric cases of CKD; however, more work will need to be done before such an approach is widely available in the clinic.
\end{abstract}

\section{CKD in children: a range of etiologies}

Chronic kidney disease (CKD) is a set of conditions characterized by gradually declining kidney function. Therapies for CKD are largely aimed at preventing progression into end-stage renal disease (ESRD). Therefore, early detection is of the utmost importance. In the US, diabetic nephropathy, vascular disease, and hypertension-attributed nephropathy are among the leading forms of CKD in adults (1). In children, the spectrum of etiologies that underlie CKD is quite different. Studies of children that have received kidney transplants for CKD that progressed to ESRD indicate that structural kidney disease accounts for roughly $30 \%$ to $40 \%$ of cases, primary glomerular injury accounts for approximately of $5 \%$ to $25 \%$ of cases (depending on age), and the remainder of cases are due to other or indeterminate causes (2).

Similar to treatment for adult cases of CKD, current therapies for established CKD in children are largely aimed at reducing the many serious complications of kidney dis- ease and slowing the progression of CKD to ESRD. Once CKD has progressed to ESRD, some form of renal replacement therapy, such as kidney transplant, hemodialysis, or peritoneal dialysis, is required for the lifetime of the individual. Because of the high morbidity associated with pediatric CKD, the best "therapy" is preventing the initial cause of disease from progressing to a chronic, irreversible state; however, such an early diagnosis or risk determination in children is often not possible.

As many cases of childhood CKD are of unknown etiology, several questions arise for the clinicians that see these patients. What studies should be included in the diagnostic evaluation of a child with idiopathic CKD? As some forms of CKD result from genetic mutations, is there a role for genetic testing in the evaluation process for children with CKD? Precision medicine, the notion that specific causes and modifiers of disease should be identified in every patient, has been touted as the future of medicine. Will new technologies allow us to pinpoint those causes that are genetic in nature? Can useful conclu-

Related Article: p. 2171

Conflict of interest: The author has declared that no conflict of interest exists.

Reference information: / Clin Invest. 2015;125(5):1799-1800. doi:10.1172/JCI81509.

sions be made about individual patients, or are genetic tools best left in the research arena as part of efforts to identify rare and common contributors to disease?

\section{Genomic imbalance: a} diagnostic tool for CKD?

A previous study by Sanna-Cherchi et al. examined the presence of duplicated or absent chromosomal segments, socalled copy number variants (CNVs), in a cohort of individuals with renal hypodysplasia, which encompasses several congenital malformations of the kidney (3). Compared with control individuals, patients with renal hypodysplasia exhibited an altered distribution of large CNVs that skewed toward larger-sized alterations. Moreover, a marked number of these alterations disrupted gene loci, compared with CNVs that were observed in control subjects.

In this issue, Verbitsky et al. (4) used a chromosomal microarray approach to evaluate the presence of CNVs in DNA from 419 children with CKD, but not syndromic disease. The subjects in this study were drawn from the Chronic Kidney Disease in Children (CKiD) cohort study, a prospective epidemiologic study designed to create a better understanding of CKD risk factors as well as the impact of declining kidney function on other aspects of health (5). Verbitsky and colleagues determined that many of these young individuals with CKD have a large excess of genomic imbalances, an observation that was not suspected after standard clinical evaluation. The presence of large genealtering CNVs was much more common in CKiD subjects than in controls $(37.7 \%$ versus $23.4 \%$ ). An increased burden of genetic alterations does not by itself allow one to make a genetic diagnosis for CKD in an individual; however, detailed annotation of the identified CNVs allowed for precise genetic diagnoses in a substantial number of cases. Specifically, 31 of the 419 individuals had a known or likely disease- 
causing genomic imbalance. As Verbitsky and colleagues illustrate, in a substantial number of these cases, the specific genetic alteration identified would be expected to lead to targeted and personalized recommendations for ongoing clinical care.

Given the rather broad and nonspecific nature of the CKD-associated phenotypes, the observation that chromosomal microarray analyses yielded informative results for individual subjects is particularly important. For any clinical test, its utility for refining a diagnosis and guiding therapy should be considered. There is little value in test results if the diagnosis is already clear before testing. If the prior probability of a positive test is so low that a false positive result is more likely than a true positive (e.g., a test result reporting, say, an apparently pathogenic polycystic kidney disease gene mutation in a healthy 80 year old with no kidney cysts), then that test is also of little value. Thus, the observation by Verbitsky and colleagues that in a collection of children with CKD, evaluation for the presence of CNVs yields a significant amount of clinically actionable information is exciting and offers a potential diagnostic test for children with idiopathic CKD.

\section{Challenges for genomic technologies in the clinic}

Should such comprehensive, genomewide analyses now be performed in all children with CKD, or should this testing be limited to selected subsets of patients? Moreover, what technologies should be used for such evaluation, and what sort of genetic alterations should be assessed? A strong case can already be made for looking for point mutations in genes known to underlie steroid-resistant nephrotic syndrome in affected children. Therapeutic decisions and recommendation for kidney transplantation may be altered by the knowledge gained from such testing (6).
But what about in the case of idiopathic CKD, in which a glomerular etiology seems less likely and the presence of genedisrupting CNVs may underlie disease? The results of the Verbitsky paper certainly provide compelling data and suggest that clinically relevant CNVs may be present in a nontrivial fraction of children with CKD.

Many of the children in the CKiD study fall into the broad category of patients with congenital abnormality of the kidney and urinary tract (CAKUT), which refers to a group of disorders that includes ureteropelvic junction obstruction, reflux nephropathy, renal hypodysplasia, and obstruction at the ureteropelvic junction. CNVs are not the only cause of CAKUT, as point mutations in a number of genes can cause CAKUT and consequent $\operatorname{CKD}(7,8)$. A comprehensive analysis of childhood CKD should likely include analyses of the sequence of these genes as well as an assessment of CNVs. Unfortunately, clinical implementation of the types of genetic analysis required to detect these abnormalities is not simple. Of course, the cost of these tests and the willingness of insurance companies to cover such analysis are distressingly complex and nonuniform across the US. The technical cost associated with performing a microarray-based analysis of patients, such as the one performed by Verbitsky et al., is no longer exorbitant. The larger obstacle for wide-scale application of this technology is the ability to perform proper quality control, analysis, and interpretation of the large data set generated. Moreover, appropriate counseling based on the results will be a potential stumbling block for this technology.

Genomic technology is rapidly changing. Whole-genome sequencing continues to become increasingly cheap and accurate. In the right setting, genome analysis not only allows for detection of structural changes, but also can identify disease- influencing and disease-causing single nucleotide changes. Until the issues of quality control and reliable data set analysis can be resolved, genome technology will not be widely available as a diagnostic tool in the clinic for CKD and other idiopathies. Nevertheless, the study by Verbitsky and colleagues suggests that for the broadly defined condition CKD, a wisely applied genetic analysis has potential to add to the precision of diagnosis and alter treatment for the better.

Address correspondence to: Martin R. Pollak, 99 Brookline Ave., Boston, Massachusetts 02214, USA. Phone: 617.667.0461; E-mail:mpollak@bidmc.harvard.edu.

1. United States Renal Data System. 2014 Annual Data Report: An overview of the epidemiology of kidney disease in the United States. Bethesda, Maryland, USA: National Institutes of Health, National Institute of Diabetes and Digestive and Kidney Diseases; 2014.

2. Smith JM, Martz K, Blydt-Hansen TD. Pediatric kidney transplant practice patterns and outcome benchmarks, 1987-2010: a report of the North American Pediatric Renal Trials and Collaborative Studies. Pediatr Transplantat. 2013;17(2):149-157.

3. Sanna-Cherchi S, et al. Copy-number disorders are a common cause of congenital kidney malformations. Am J Hum Genet. 2012;91(6):987-997.

4. Verbitsky M, et al. Genomic imbalances in pediatric patients with chronic kidney disease. J Clin Invest. 2015;125(5):2171-2178.

5. Wong CJ, Moxey-Mims M, Jerry-Fluker J, Warady BA, Furth SL. CKiD (CKD in children) prospective cohort study: a review of current findings. Am J Kidney Dis. 2012;60(6):1002-1011.

6. Sadowski CE, et al. A single-gene cause in $29.5 \%$ of cases of steroid-resistant nephrotic syndrome [published online ahead of print October 27, 2014]. JAm Soc Nephrol. doi:10.1681/ASN.2014050489.

7. Vivante A, Kohl S, Hwang DY, Dworschak GC, Hildebrandt F. Single-gene causes of congenital anomalies of the kidney and urinary tract (CAKUT) in humans. Pediatr Nephrol. 2014;29(4):695-704.

8. Hwang DY, et al. Mutations in 12 known dominant disease-causing genes clarify many congenital anomalies of the kidney and urinary tract. Kidney Int. 2014;85(6):1429-1433. 